\title{
Log-periodic dipole antenna with low cross-polarization
}

\author{
Pivnenko, Sergey
}

Published in:

Proceedings of the European Conference on Antennas and Propagation

Link to article, DOI:

10.1109/EUCAP.2006.4585044

Publication date:

2006

Document Version

Publisher's PDF, also known as Version of record

Link back to DTU Orbit

Citation (APA):

Pivnenko, S. (2006). Log-periodic dipole antenna with low cross-polarization. In Proceedings of the European Conference on Antennas and Propagation (pp. 1-4). IEEE. https://doi.org/10.1109/EUCAP.2006.4585044

\section{General rights}

Copyright and moral rights for the publications made accessible in the public portal are retained by the authors and/or other copyright owners and it is a condition of accessing publications that users recognise and abide by the legal requirements associated with these rights.

- Users may download and print one copy of any publication from the public portal for the purpose of private study or research.

- You may not further distribute the material or use it for any profit-making activity or commercial gain

- You may freely distribute the URL identifying the publication in the public portal

If you believe that this document breaches copyright please contact us providing details, and we will remove access to the work immediately and investigate your claim. 


\title{
LOG-PERIODIC DIPOLE ANTENNA WITH LOW CROSS-POLARIZATION
}

\author{
Sergey Pivnenko \\ Ørsted-DTU, Technical University of Denmark \\ Ørsteds Plads, Bldg. 348, DK-2800 Kgs. Lyngby, Denmark \\ Email: sp@oersted.dtu.dk
}

\begin{abstract}
In this work, log-periodic antennas with improved cross-polarization level were studied. It was found that some modifications of the traditional design lead to an essential improvement of the co-to-cross polarization ratio up to $40 \mathrm{~dB}$. An improved design of a log-periodic dipole antenna with low cross-polarization level is described. Some recommendations regarding improvement of the polarization characteristics of logperiodic antennas in general are also given. It was also found that log-periodic antennas can be attributed to the class of so-called first-order $(\mathrm{m}= \pm 1)$ antennas, which is an important requirement for probes in spherical nearfield antenna measurements.
\end{abstract}

\section{INTRODUCTION}

The log-periodic principle was established in the late 1950s and gave rise to the development of several types of wideband antennas [1]. Different log-periodic antennas are now used in many applications where wide bandwidth is one of the important requirements. However, there is a drawback in most of these antennas, since the cross-polarization level is not acceptable for certain applications. For example, typical co-polar to cross-polar ratio of commercially available log-periodic antennas is about $25 \mathrm{~dB}$, but for some applications larger values are required.

One such application is near-field antenna measurements, where a relatively narrow-band, less than an octave, open-ended waveguide is often used as a probe. Several probes are thus necessary to cover a wide frequency range and their interchange is unavoidable if the measurement frequency is to be changed. A wideband probe covering the frequency range, e.g. from 2-18 GHz, would simplify the manipulations and speedup the measurements. The requirements for the crosspolarization level of the near-field probes are quite high and the available log-periodic antennas cannot be applied here.

The log-periodic antennas were studied in this work with the aim to improve their cross-polarization level. It was found that some modifications of the traditional design lead to an essential improvement of the co-tocross polarization ratio, particularly, the values better than $40 \mathrm{~dB}$ were obtained. The purpose of this paper is to introduce an improved design of a log-periodic dipole antenna with low cross-polarization level. Some recommendations regarding improvement of the polarization characteristics of log-periodic antennas in general are also given.

During the study, it was also found that the log-periodic antennas can be attributed to the class of so-called firstorder $(\mathrm{m}= \pm 1)$ antennas, which is an important requirement for probes in spherical near-field antenna measurements [2]. This requirement implies that the radiation pattern of the probe has only first-order $(\sin \phi$, $\cos \phi)$ azimuthal dependence and thus the complete information about the pattern can be obtained from measurements of only two orthogonal cuts. The logperiodic dipole antennas do not satisfy this requirement completely since their spectra contain also some power in the spherical modes with azimuthal indices $m \neq \pm 1$, but their level is relatively low.

\section{IMPROVED DESIGN}

A typical design of the log-periodic dipole antenna implies alternating vertical displacement of dipole arms to ensure their proper excitation by the feed line. This is illustrated in Fig. 1. A zoomed-in view also shows the cross-section of the region around the feed line for illustration of displacement of the dipoles.

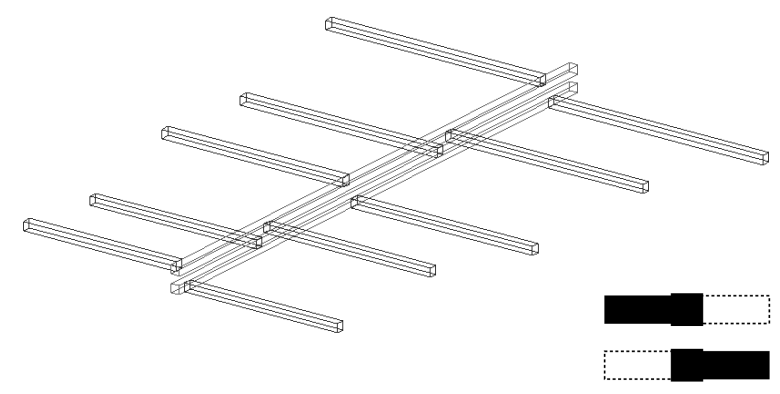

Figure 1. Typical design of a log-periodic dipole antenna.

A simulation of a log-periodic dipole antenna of a typical design with 5 dipoles has been carried out with the HFSS software to check if such a displacement produces an increased level of cross-polarized field. The design was made for the frequency range from 1.5-2.0 
GHz. The vertical displacement of the centers of the two arms of each dipole was approximately $1 / 10$ of the arm length. The simulated co-polar and cross-polar patterns (according to Ludwig $3^{\text {rd }}$ definition) in the E- and $\mathrm{H}$ planes are presented in Fig. 2 for the frequency 1.6 $\mathrm{GHz}$. It is seen that the cross-polarized field level relative to the peak directivity value is about $-26 \mathrm{~dB}$, which also holds at other frequencies of the design range.

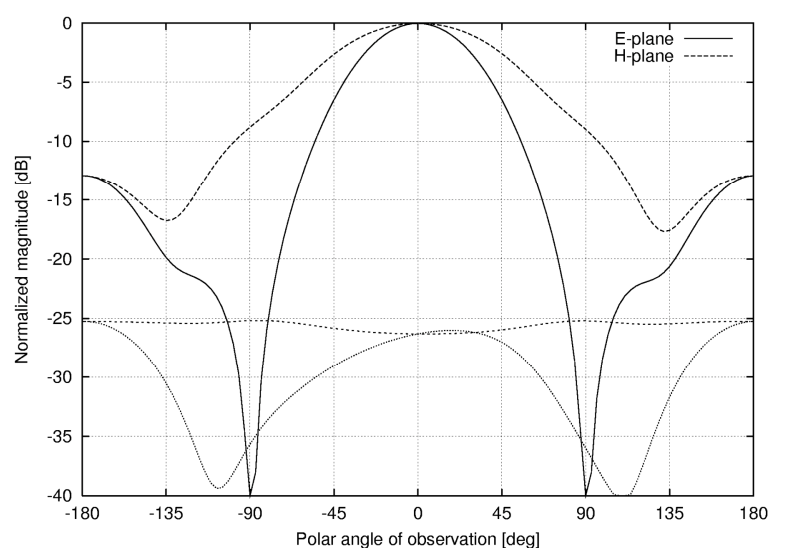

Fig. 2. Simulated radiation pattern of a log-periodic dipole antenna of typical design.

It was supposed that the cross-polarized field is due to the displacement of the dipole arms and an alternative design of the antenna was then simulated in which the displacement of the dipole arms is significantly reduced, see Fig. 3. In this case, the vertical displacement of the centers of the two arms of each dipole was approximately $1 / 100$ of the arm length. Note that in the improved design the feed line is changed from two wires of square cross-section to the two strips as shown in the zoomed-in view of the cross-section of the feed line region in Fig. 3.

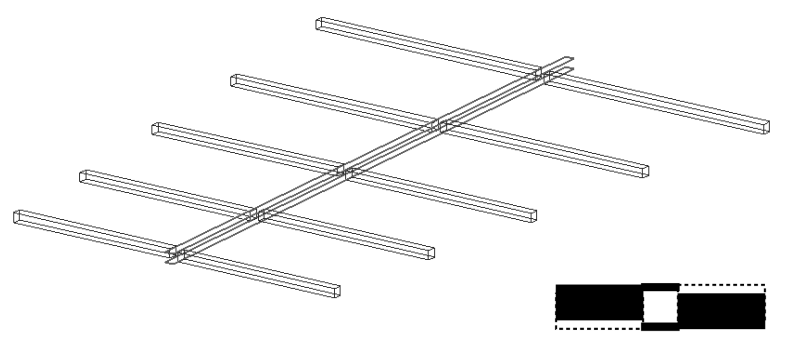

Fig. 3. An improved design of a log-periodic dipole antenna.

The simulated co-polar and cross-polar patterns in the E-and H-planes for the improved design of the antenna are presented in Fig. 4. It is seen that the cross-polarized field is reduced to the level of about $-37 \mathrm{~dB}$ relative to the peak value. At the same time, the changes in the copolar pattern are very small, as it could be anticipated.

It became clear that reduction of the vertical displacement of the two arms of each dipole results in a significant reduction of the cross-polarized field. Therefore, a desirable low level of cross-polarized field of log-periodic antennas could be obtained by placing the two arms of each dipole avoiding the vertical displacement and preferably in the same plane.

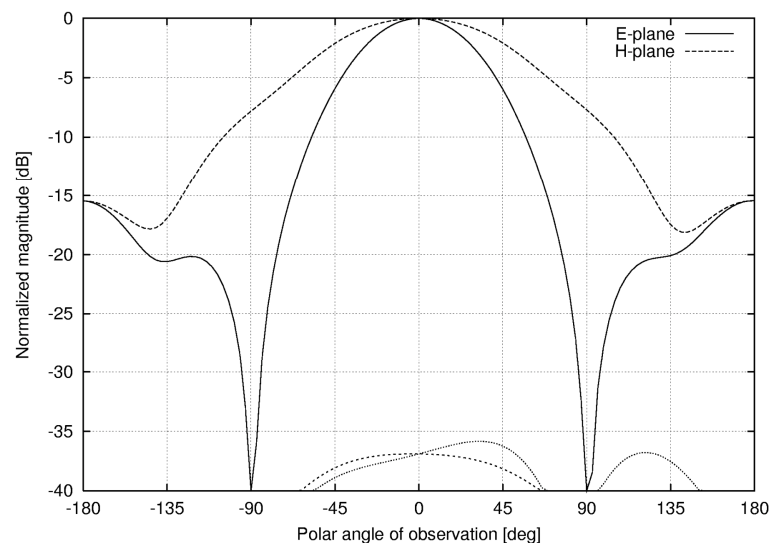

Fig. 4. Simulated radiation pattern of a log-periodic dipole antenna of improved design.

\section{EXPERIMENTAL VERIFICATION}

In order to check the conclusions made above and verify experimentally the results of the simulations, a prototype log-periodic dipole antenna with 9 dipoles was manufactured using printed circuit technology on FR4 substrate. The antenna was designed for the frequency range $1.5-2.0 \mathrm{GHz}$ with the nominal directivity of $10 \mathrm{dBi}$. The photo of the manufactured prototype antenna is shown in Fig. 5.

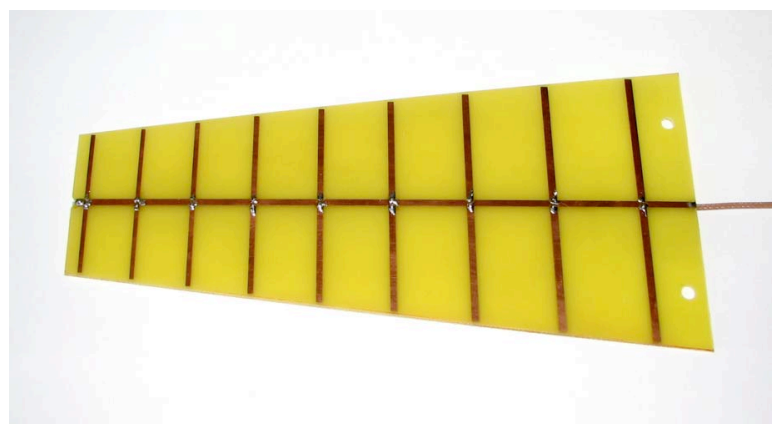

Fig. 5. Photo of the manufactured prototype antenna.

A particular feature of the manufactured prototype antenna is illustrated in Fig. 6. It is seen that each dipole arm consists of two strips located on both sides of the 
substrate and connected together by vias at the feed line. This ensures symmetric excitation of both strips of the dipole arm as well as symmetric location of the arms with respect to the feed line without their vertical displacement.

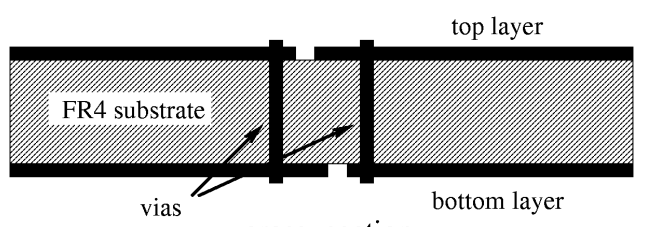

cross-section

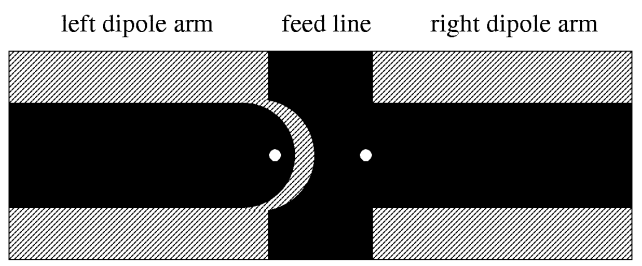

top view

Fig. 6. Region around feed line showing the features of the symmetric dipole arm feeding.

The radiation pattern of the manufactured prototype antenna was measured at the DTU-ESA Spherical NearField Antenna Test Facility at 10 frequencies in the range from 1.5-1.95 GHz. The measured co-polar and cross-polar patterns in the $\mathrm{E}$ - and $\mathrm{H}$ planes are presented in Fig. 7 for the frequency $1.7 \mathrm{GHz}$.

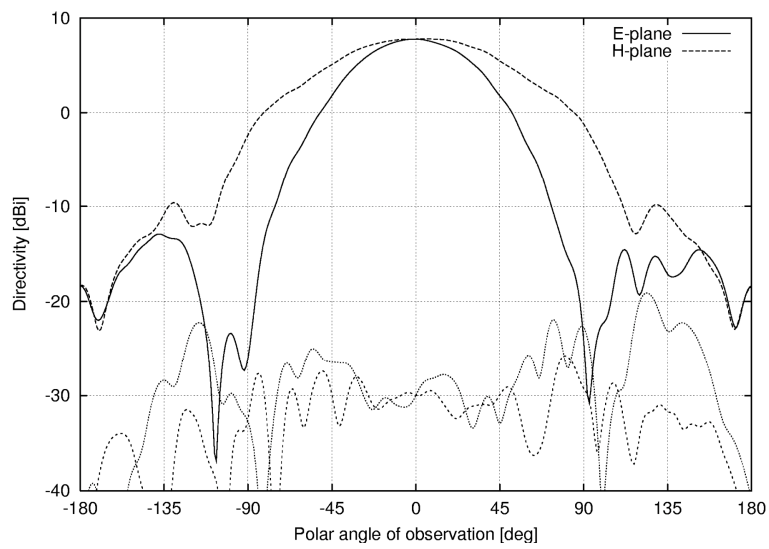

Fig. 7. Measured radiation pattern of the manufactured prototype of the log-periodic dipole antenna at $1.7 \mathrm{GHz}$.

It is seen from Fig. 7 that the measured pattern is almost symmetric with a very low level of the cross-polarized field. The measured directivity of the antenna is, however, less than the design goal of $10 \mathrm{~dB}$, which is the case for all frequencies. The measured directivity values vary from $8.8 \mathrm{~dB}$ at the lowest frequency to 6.8
$\mathrm{dB}$ at the highest frequency. It is noted that the permittivity of the substrate was not taken into account in designing the antenna, which may cause the observed reduced directivity values.

The polarization characteristics of the manufactured prototype antenna were measured with the three-antenna measurement technique at 20 frequencies in the same frequency range. The polarization axial ratio versus frequency is shown in Fig. 8.

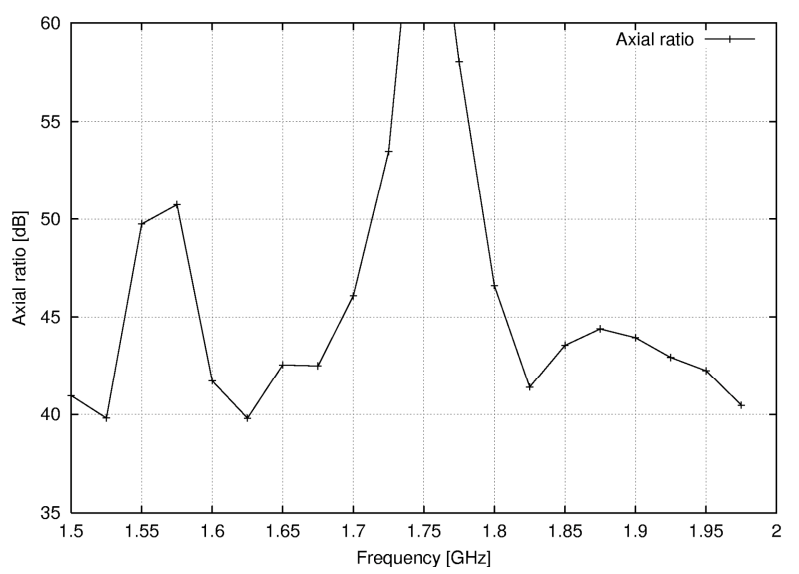

Fig. 8. Measured axial ratio of the manufactured prototype of the log-periodic dipole antenna.

Figure 8 shows that the axial ratio of the manufactured prototype antenna is quite high, it is above $40 \mathrm{~dB}$ level with some oscillations from about $40 \mathrm{~dB}$ to more than $60 \mathrm{~dB}$ in the measured frequency range. These values prove the expected low level of the cross-polarized field. The measured polarization tilt angle varies from about $-0.2^{\circ}$ at the lowest frequency to about $1.2^{\circ}$ at the highest frequency. At $1.7 \mathrm{GHz}$ it is about $0.4^{\circ}$, which explains the difference between $46 \mathrm{~dB}$ axial ratio in Fig. 8 and about $38 \mathrm{~dB}$ co-to-cross-polar ratio in Fig. 7.

\section{SPECTRUM OF SPHERICAL MODES}

An antenna to be used as a probe in spherical near-field antenna measurements should posses a certain property related to its radiation pattern. The radiation pattern should have only first-order $(\sin \phi, \cos \phi)$ azimuthal dependence. As a result of this feature the spectrum of spherical modes of such pattern contains only the modes with azimuthal indices $m= \pm 1$. This requirement is set by the presently available probe correction procedure for the spherical near-field antenna measurements [2].

The measured pattern of the manufactured prototype antenna was checked for conformance to this requirement. The spectrum of the spherical modes of the measured pattern at $1.7 \mathrm{GHz}$ is shown in Fig. 9. The discrete values are connected by straight lines. Similar 
spectra were also obtained from the pattern measured at the other frequencies in the range from 1.5-1.95 GHz.

The spectrum of a conical horn antenna excited by a dominant circular waveguide mode, which is a good $\mathrm{m}= \pm 1$ antenna, is also given in Fig. 9 for comparison. This spectrum was obtained from the measured radiation pattern of the conical horn.

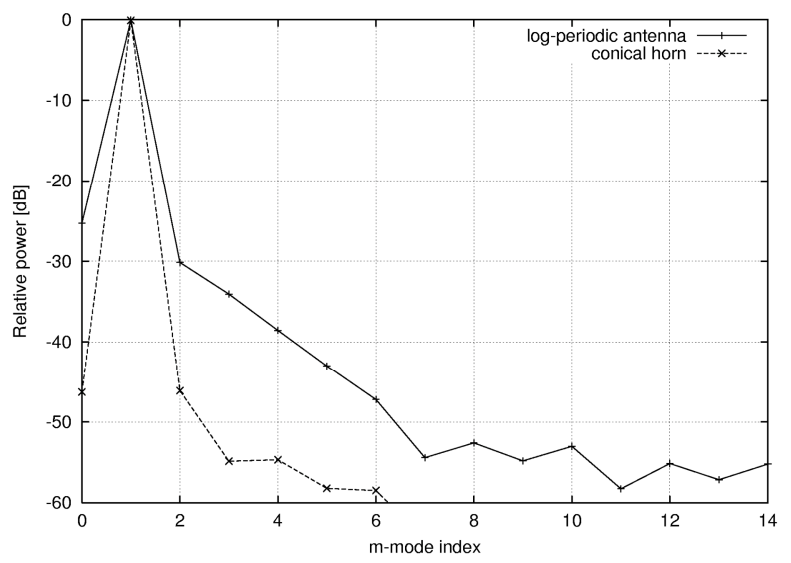

Fig. 9. Spectrum of azimuthal spherical modes of the log-periodic dipole antenna and a conical horn.

It is seen that the radiated power of the log-periodic antenna is contained mostly in the azimuthal modes with indices $m= \pm 1$, while the modes with other indices are quite low, at the level of approximately $-25 \mathrm{~dB}$ to $-30 \mathrm{~dB}$ and lower. It is however noted that these modes $(m \neq \pm 1)$ are not as low as for the conical horn antenna, for which the typical level of the modes with indices $\mathrm{m} \neq \pm 1$ is below $-45 \mathrm{~dB}$.

The significance of the modes with $m \neq \pm 1$ for the logperiodic antenna, if it is applied as probe in spherical near-field antenna measurements with the available first-order probe correction procedure, is a topic of a future study.

Alternatively, the influence of these modes can be compensated by application of a recently developed iterative probe correction technique [3], which is well suited for non-ideal first-order probes.

\section{CONCLUSIONS}

The log-periodic antennas were studied in this work with the aim to improve their cross-polarization level. It was found that some modifications of the traditional design lead to an essential improvement of the co-tocross polarization ratio, particularly, the values better than $40 \mathrm{~dB}$ were obtained. An improved design of a logperiodic dipole antenna with a very low crosspolarization level was introduced. Some recommendations regarding improvement of the polarization characteristics of log-periodic antennas in general were also given. It was also found that the logperiodic antennas can be preliminary attributed to the class of so-called first-order $(\mathrm{m}= \pm 1)$ antennas, which is an important requirement for probes in spherical nearfield antenna measurements.

\section{REFERENCES}

1. C. E. Smith (Ed.), Log Periodic Antenna Design Handbook, Smith Electronics, Inc., Cleveland, USA, 1966.

2. J. E. Hansen (ed.), Spherical Near-Field Antenna Measurements, Peter Peregrinus, Ltd., London, 1988.

3. T. Laitinen, S. Pivnenko, O. Breinbjerg, "Iterative probe correction technique for spherical near-field antenna measurements," IEEE Antennas and Wireless Propagation Letters, Vol. 4, 2005, pp. 221223. 